\title{
Potencial de uso de espécies de pinus tropicais para produção de painéis cimento-madeira
}

\author{
Potential use of tropical pinus species \\ for wood cement panel production
}

\author{
Setsuo Iwakiri' ${ }^{1}$, Rosilani Trianoski ${ }^{1}$, Ana Paula Namikata da Fonte ${ }^{2}$, \\ Débora Jordão Cezimbra², Igor Mottinha Fomin², Richard Molleken²
}

\begin{abstract}
Resumo
Este trabalho teve como objetivo avaliar o potencial de utilização da madeira de quatro espécies de pinus tropicais para produção de painéis cimento-madeira. As espécies avaliadas foram: Pinus caribaea, Pinus maximinioi, Pinus oocarpa e Pinus tecunumanii. O Pinus taeda foi utilizado como testemunha. Foram produzidos painéis experimentais com densidade nominal de $1,20 \mathrm{~g} / \mathrm{cm}^{3}$ e relações madeira:cimento de 1:2,5 e 1:3,0. Os painéis foram prensados com pressão específica de $4 \mathrm{MPa}$ a temperatura ambiente, tempo de prensagem / grampeamento de 24 horas e tempo de maturação de 28 dias. A qualidade dos painéis foi avaliada por meio de ensaios de propriedades físicas e mecânicas de acordo com a norma Europeia CEN. A relação madeira:cimento não afetou de forma significativa as propriedades dos painéis. Com base nas avaliações coMParativas com os resultados obtidos para a espécie testemunha e dados apresentados na literatura, pode-se afirmar que as quatro espécies de pinus tropicais são potencialmente viáveis para produção de painéis cimento-madeira.
\end{abstract}

Palavras-chave: aglutinante mineral, construção civil, florestas plantadas.

\begin{abstract}
This study aimed to evaluate the potential use of wood of four tropical pine species for the production of wood-cement panels. The species were Pinus caribaea, Pinus maximinioi, Pinus oocarpa and Pinus tecunumanii. Pinus taeda was used as control. Experimental panels were produced with a density of 1.20 $\mathrm{g} / \mathrm{cm}^{3}$ and wood: cement ratio of $1: 2.5$ and 1:3.0. The panels were pressed with a specific pressure of $4 \mathrm{MPa}$ at room temperature, pressing time / Clipping of 24 hours and maturing time of 28 days. The quality of the panels was evaluated by testing the physical and mechanical properties according to the European standard CEN. The wood: cement ratio did not affect significantly the properties of the panels. Based on comparative evaluations with the values obtained for the control species and data presented in the literature, it can be said that the four tropical pine species are potentially usable for the production of cement-wood panels.
\end{abstract}

Keywords: mineral binder, civil construction, forest plantation.

\section{INTRODUÇÃO}

Os painéis cimento-madeira são produtos constituídos de partículas de madeira com um aglutinante mineral e consolidados por meio de prensagem à temperatura ambiente. São amplamente empregados na construção civil, principalmente em países asiáticos e europeus, devido às suas características vantajosas como relatado por Moslemi (1974): (i) são produtos virtualmente incombustíveis; (ii) apresentam alta resistência mecânica e à umidade; (iii) são resistentes ao ataque de agentes biodegradadores; e, (iv) são isolantes térmicos e acústicos.

No Brasil, os painéis cimento-madeira não são produzidos comercialmente, entretanto, apresentam grande potencial para a sua introdução, tendo em vista a necessidade do setor de construção civil por materiais alternativos de baixo custo para viabilizar a produção de habitações em larga escala. Outros fatores positivos que podem viabilizar a produção de painéis cimento-madeira no

${ }_{1}^{1}$ Professor(a) Titular do Departamento de Engenharia e Tecnologia Florestal. UFPR - Universidade Federal do Paraná / Setor e Ciências Agrárias. Av. Lothário Meissner, 3400 - Jardim Botânico - 80210170 - Curitiba, PR, Brasil. E-mail: setsuo.ufpr@gmail.com; rosilani@ufpr.br

${ }^{2}$ Mestranda do Programa de Pós-Graduação em Engenharia Florestal. UFPR - Universidade Federal do Paraná / Setor e Ciências Agrárias. Av. Lothário Meissner, 3400 - Jardim Botânico - 80210170 - Curitiba, PR, Brasil. E-mail: ana.namikata@gmail.com; deborajordao@hotmail.com; fomin@ufpr.br; richard molleken@hotmail.com

Sci. For., Piracicaba, v. 45, n. 113, p. 151-159, mar. 2017 DOI: dx.doi.org/10.18671/scifor.v45n113.15 
Brasil é a grande disponibilidade de madeiras na forma de resíduos de processamento industrial e autossuficiência do país na produção do cimento Portland.

Pesquisas sobre painéis cimento-madeira têm sido realizadas ao nível de laboratório no Brasil, utilizando espécies provenientes de plantios florestais. Parchen et al. (2015) avaliaram a qualidade de painéis cimento-madeira de baixa densidade produzidos com madeiras provenientes de resíduos de galhos e troncos e prensados por método de vibro-compactação; Iwakiri et al. (2015) aplicaram diferentes métodos de tratamentos de partículas de madeira de Eucalyptus benthamii na produção de painéis cimento-madeira; Castro et al. (2015) avaliaram os efeitos de diferentes aditivos químicos e métodos de formação dos painéis cimento-madeira nas suas propriedades; Iwakiri e Prata (2008) e Silva et al. (2005) avaliaram as propriedades físicas e mecânicas dos painéis cimento-madeira produzidos com madeiras de Eucalyptus grandis, Eucalyptus dunnii e Eucalyptus urophylla. Os referidos pesquisadores obtiveram resultados satisfatórios nas avaliações das propriedades dos painéis produzidos com diferentes espécies e parâmetros de processo.

Dentre os fatores que influenciam no processo produtivo de painéis cimento-madeira, destaca-se a espécie, principalmente no que se refere à composição química da madeira. Segundo Simatupang et al. (1978), os extrativos presentes na madeira são os principais responsáveis pela inibição da cura e endurecimento do cimento, sendo seus princípios ativos os compostos fenólicos e os carboidratos livres. Os autores afirmam também que as espécies de folhosas tropicais apresentam influência negativa mais pronunciada na cura e endurecimento do cimento do que as de coníferas. Beraldo et al. (2002) citam que a presença de açucares e extrativos solúveis em água ou álcali retarda o tempo de pega do cimento. Para minimizar os efeitos inibidores de extrativos presentes na madeira de algumas espécies na cura do cimento, Moslemi et al. (1983) recomendam três métodos de tratamentos de partículas: imersão em água fria por 24 horas, imersão em água quente por seis horas, e imersão em solução de $\mathrm{NaOH}(1 \%)$ por duas horas, seguida de boa lavagem para remoção de extrativos.

Além da madeira, outros parâmetros importantes no processo de produção de painéis cimento-madeira são a relação madeira:cimento, a relação água:cimento, o tipo e quantidade de acelerador de cura. Segundo Latorraca (2000), a relação madeira:cimento mais empregada industrialmente é de 1:2,75. Simatupang et al. (1978) relatam que, para manufatura de painéis cimento-madeira de alta massa específica, é utilizada uma proporção de água em relação ao cimento de 0,4 . Os aditivos químicos são empregados com propósito de reduzir o tempo de endurecimento do cimento, acelerando a sua cura. Os sais como cloreto de cálcio, cloreto de magnésio, silicato de sódio e silicato de potássio, são alguns dos aditivos utilizados, sendo que o mais empregado é o cloreto de cálcio. Moslemi et al. (1983) e Rashwan et al. (1992) recomendam como quantidades ideais de acelerador de cura no processo produtivo, proporções de 3 a 5\% de cloreto de cálcio em relação ao peso do cimento.

Tendo em vista a importância de desenvolvimento de novos materiais para a construção civil e, da necessidade de uso de espécies alternativas de rápido crescimento para uso industrial, esta pesquisa teve como objetivo avaliar o potencial de uso de espécies de pinus tropicais para produção de painéis cimento-madeira.

\section{MATERIAL E MÉTODOS}

Foram utilizadas nesta pesquisa madeiras de Pinus maximinoi, Pinus tecunumanii, Pinus oocarpa, Pinus caribaea var. hondurensis e Pinus taeda, com 18 anos de idade, provenientes de plantios florestais localizados na região de Itararé - SP e Ventania - PR. O cimento Portland foi utilizado como aglutinante mineral e o cloreto de cálcio como acelerador de cura, sendo ambos adquiridos no mercado local.

O material foi obtido na forma de tábuas com 25,4 mm de espessura, as quais foram seccionadas em blocos com dimensões compatíveis com o gerador de partículas e mantidas submersas em água por um período de 15 dias. As partículas foram obtidas com espessura nominal de 0,7 mm, secas ao teor de umidade entre 15 a $20 \%$ e reprocessadas no moinho de martelo para redução nas suas dimensões. A seguir, as partículas foram peneiradas para retirada dos "finos".

O plano experimental consistiu em cinco espécies e duas relações madeira:cimento de $1: 2,5 \mathrm{e}$ $1: 3,0$. O Pinus taeda foi utilizado como testemunha, tendo em vista ser a espécie comercial amplamente empregada pelas indústrias. 
Para a produção de painéis experimentais, procedeu-se inicialmente o cálculo de cada um dos componentes da mistura: partículas de madeira, cimento, água e cloreto de cálcio. As proporções de cada componente na mistura foram as seguintes: relação madeira:cimento de 1:2,5 e 1:3,0; fator água:cimento de 0,4 e 3\% de cloreto de cálcio em relação ao peso do cimento. Os cálculos foram realizados para massa específica nominal do painel de $1,20 \mathrm{~g} / \mathrm{cm}^{3}$. Devidamente pesados, os componentes foram misturados numa betoneira para obtenção de uma massa homogênea.

A massa de cada painel, composta pelas quatro matérias-primas, foi pesada e distribuída aleatoriamente na caixa formadora do colchão, com dimensões de $385 \mathrm{~mm}$ de largura e $505 \mathrm{~mm}$ de comprimento. Abaixo e acima do colchão de partículas foram colocadas placas de alumínio untadas com óleo mineral para facilitar o desmolde após prensagem e duas barras de ferro de $15 \mathrm{~mm}$ para controle da espessura do painel.

O colchão foi prensado à temperatura ambiente com pressão específica de $40 \mathrm{kgf} / \mathrm{cm}^{2}$, com três painéis sobrepostos em sequência e mantidos sob restrição no aparato de grampeamento por período de 24 horas para o endurecimento. Após esse período, os grampos foram retirados e os painéis foram acondicionados na câmara climática à temperatura de $20 \pm 2{ }^{\circ} \mathrm{C}$ e umidade relativa de $65 \pm$ $5 \%$, por um período de 28 dias para completa maturação.

Para avaliação das propriedades físicas e mecânicas, foram retirados de cada painel, seis corpos-de-prova para ensaios de massa específica aparente, absorção de água e inchamento em espessura após 2 e 24 horas de imersão em água, seis corpos-de-prova para tração perpendicular (ligação interna) e seis corpos-de-prova para flexão estática (módulo de elasticidade e módulo de ruptura). Os ensaios foram realizados de acordo com os procedimentos descritos nas normas EN 317 (EN,1993a), EN 319 (EN,1993b) e EN 310 (EN,1993c), respectivamente. Na Figura 1 está apresentado o esquema de corte dos corpos-de-prova para os ensaios.

\begin{tabular}{|c|c|c|c|c|c|c|c|}
\hline \multirow[t]{6}{*}{$1 a$} & \multirow[t]{6}{*}{$2 a$} & $3 a$ & \multirow[t]{6}{*}{$4 a$} & $5 a$ & \multirow[t]{6}{*}{$6 a$} & \multirow[t]{6}{*}{$7 a$} & \multirow[t]{6}{*}{$8 a$} \\
\hline & & $3 a$ & & $5 a$ & & & \\
\hline & & $3 a$ & & $5 a$ & & & \\
\hline & & $3 a$ & & $5 a$ & & & \\
\hline & & $3 a$ & & $5 a$ & & & \\
\hline & & $3 a$ & & $5 a$ & & & \\
\hline
\end{tabular}

la, 2a, 4a, 6a, 7a, 8a: flexão estática; 3a: massa específica aparente, absorção de água e inchamento em espessura; 5a: tração perpendicular. Figura 1. Esquema de corte dos corpos-de-prova para ensaios.

Figure 1. Cutting scheme of specimens for testing.

Os resultados de ensaios foram analisados por meio de ANOVA e teste de Tukey para coMParações de médias ao nível de probabilidade de $95 \%$. O delineamento empregado foi o fatorial 5 × 2 , para espécies e relações madeira:cimento, respectivamente.

\section{RESULTADOS E DISCUSSÃO}

\section{Propriedades físicas dos painéis}

Na Tabela 1 estão apresentados os resultados de massa específica aparente, absorção de água e inchamento em espessura após 2 e 24 horas de imersão.

Os valores médios de massa específica aparente dos painéis variaram na faixa de 1,14 a 1,27 g/ $\mathrm{cm}^{3}$, estando próximos da densidade nominal calculada de $1,20 \mathrm{~g} / \mathrm{cm}^{3}$. Os baixos valores dos coeficientes de variação indicam que os procedimentos operacionais para a manufatura de painéis ao nível de laboratório foram adequados.

Os resultados de absorção de água e inchamento em espessura indicam que houve diferenças significativas entre as médias dos tratamentos, tanto para 2 horas, quanto para 24 horas de imersão em água. 
Iwakiri et al. - Potencial de uso de espécies de pinus tropicais para produção de painéis cimento-madeira

Tabela 1. Resultados de massa específica aparente, absorção de água e inchamento em espessura.

Table 1. Results of density, water absorption and thickness swelling.

\begin{tabular}{|c|c|c|c|c|c|}
\hline Tratamento & ME $\left(\mathrm{g} / \mathrm{cm}^{3}\right)$ & AA 2 h (\%) & AA 24h (\%) & IE 2h (\%) & IE 24h (\%) \\
\hline P. taeda $1: 2,5$ & $\begin{array}{c}1,20 \\
(2,04) \\
\end{array}$ & $\begin{array}{c}7,00 \mathrm{a} \\
(23,35) \\
\end{array}$ & $\begin{array}{c}12,45 \mathrm{ab} \\
(14,71) \\
\end{array}$ & $\begin{array}{c}0,66 \text { a } \\
(37,49) \\
\end{array}$ & $\begin{array}{c}1,18 a \\
(26,24)\end{array}$ \\
\hline P. taeda $1: 3,0$ & $\begin{array}{c}1,26 \\
(2,87) \\
\end{array}$ & $\begin{array}{l}7,59 \mathrm{ab} \\
(22,56)\end{array}$ & $\begin{array}{c}12,12 \mathrm{ab} \\
(14,51)\end{array}$ & $\begin{array}{l}0,54 \mathrm{ab} \\
(36,62)\end{array}$ & $\begin{array}{l}0,78 \text { bc } \\
(40,27)\end{array}$ \\
\hline P. maximinoi 1:2,5 & $\begin{array}{c}1,15 \\
(5,36)\end{array}$ & $\begin{array}{c}9,34 \mathrm{c} \\
(20,06)\end{array}$ & $\begin{array}{c}13,65 \mathrm{bc} \\
(14,32)\end{array}$ & $\begin{array}{c}0,47 \mathrm{abc} \\
(44,98)\end{array}$ & $\begin{array}{c}0,85 \mathrm{abc} \\
(36,18)\end{array}$ \\
\hline P. maximinoi 1:3,0 & $\begin{array}{c}1,25 \\
(3,21)\end{array}$ & $\begin{array}{c}7,44 \mathrm{a} \\
(22,76) \\
\end{array}$ & $\begin{array}{l}11,32 \mathrm{a} \\
(15,59)\end{array}$ & $\begin{array}{l}0,40 \mathrm{bc} \\
(62,29)\end{array}$ & $\begin{array}{c}0,71 \mathrm{c} \\
(55,48)\end{array}$ \\
\hline P. tecunumanii $1: 2,5$ & $\begin{array}{c}1,18 \\
(6,65)\end{array}$ & $\begin{array}{l}7,22 \mathrm{a} \\
(22,91)\end{array}$ & $\begin{array}{c}11,93 \mathrm{ab} \\
(16,42)\end{array}$ & $\begin{array}{c}0,44 \mathrm{abc} \\
(60,26)\end{array}$ & $\begin{array}{l}0,77 \mathrm{bc} \\
(47,43)\end{array}$ \\
\hline P. tecunumanii $1: 3,0$ & $\begin{array}{l}1,275 \\
(5,85)\end{array}$ & $\begin{array}{c}9,11 \mathrm{c} \\
(19,88)\end{array}$ & $\begin{array}{c}12,78 \mathrm{ab} \\
(15,94)\end{array}$ & $\begin{array}{c}0,44 \mathrm{abc} \\
(66,21)\end{array}$ & $\begin{array}{l}0,76 \mathrm{bc} \\
(47,69)\end{array}$ \\
\hline P. oocarpa 1:2,5 & $\begin{array}{c}1,14 \\
(2,57)\end{array}$ & $\begin{array}{l}8,81 \mathrm{bc} \\
(28,94)\end{array}$ & $\begin{array}{l}14,51 \mathrm{c} \\
(20,78)\end{array}$ & $\begin{array}{c}0,46 \mathrm{abc} \\
(53,63)\end{array}$ & $\begin{array}{l}0,71 \mathrm{~cd} \\
(39,77)\end{array}$ \\
\hline P. oocarpa 1:3,0 & $\begin{array}{l}1,203 \\
(2,50)\end{array}$ & $\begin{array}{c}8,30 \mathrm{abc} \\
(23,48)\end{array}$ & $\begin{array}{c}13,20 \mathrm{bc} \\
(15,59)\end{array}$ & $\begin{array}{c}0,29 \mathrm{c} \\
(63,11)\end{array}$ & $\begin{array}{c}0,42 \mathrm{c} \\
(64,45)\end{array}$ \\
\hline P. caribaea $1: 2,5$ & $\begin{array}{c}1,19 \\
(5,36) \\
\end{array}$ & $\begin{array}{c}7,92 \mathrm{abc} \\
(10,05)\end{array}$ & $\begin{array}{c}12,39 a b \\
(7,97) \\
\end{array}$ & $\begin{array}{l}0,62 \mathrm{ab} \\
(53,24)\end{array}$ & $\begin{array}{l}1,04 a b \\
(38,60)\end{array}$ \\
\hline P. caribaea $1: 3,0$ & $\begin{array}{c}1,21 \\
(5,50)\end{array}$ & $\begin{array}{c}8,06 \text { abc } \\
(19,15)\end{array}$ & $\begin{array}{c}12,05 \mathrm{ab} \\
(18,41)\end{array}$ & $\begin{array}{c}0,46 \mathrm{abc} \\
(58,05)\end{array}$ & $\begin{array}{l}0,70 \mathrm{~cd} \\
(53,73)\end{array}$ \\
\hline
\end{tabular}

ME: massa específica aparente; AA: absorção de água; IE: inchamento em espessura; Médias seguidas de mesma letra na mesma coluna são estatisticamente iguais ao nível de probabilidade de $95 \%$. Valores entre parênteses referem-se ao coeficiente de variação.

Para absorção de água 2 horas, os valores médios variaram de 7,00\% a 9,34\%. Os painéis de $P$. taeda produzidos com relação madeira;cimento de 1:2,5 apresentaram médias estatisticamente iguais em comparação aos painéis de $P$. taeda com relação 1:3,0, P. maximinoi com 1:3,0, P. tecunumanii com 1:2,5, P. oocarpa com 1:3,0, P. caribaea com 1,25 e 1:3,0 e, estatisticamente inferiores em comparação aos demais tratamentos.

Já, para absorção de água 24 horas, os valores médios variaram de 11,32\% a 14,51\%. Os painéis de P. maximinoi produzidos com relação madeira:cimento de 1:3,0 apresentaram médias estatisticamente iguais em comparação aos painéis de $P$. taeda com relação 1:2,5 e 1:3,0, P. tecunumanii com 1:2,5 e 1:3,0, $P$. caribaea com 1:2,5 e 1:3,0 e, estatisticamente inferiores em comparação aos demais tratamentos.

Os resultados de absorção de água 24 horas obtidos nesta pesquisa foram satisfatórios em comparação aos apresentados na literatura por alguns pesquisadores. Iwakiri et al. (2012) encontraram para painéis cimento-madeira de Schizolobium amazonicum e Cecropia hololeuca, valores de absorção de água na faixa de $23,22 \%$ a $25,27 \%$ e de $17,64 \%$ a $20,86 \%$, respectivamente; Iwakiri e Prata (2008) encontraram para painéis cimento-madeira de Eucalyptus grandis Hill ex Maiden e Eucalyptus dunnii Maiden, valores de absorção de água na faixa de $15,15 \%$ a 20,34\% e de $14,27 \%$ a 20,30\%, respectivamente; Okino et . al. (2004) encontraram para painéis cimento-madeira de Hevea brasiliensis valores de absorção de água 24 horas variando de 15,70\% a 18,50\%. Cabe ressaltar que, o maior valor de absorção de água 24 horas obtido para quatro espécies de pinus tropicais foi de $14,51 \%$, sendo, portanto, inferiores aos valores apresentados por referidos pesquisadores.

Com relação ao inchamento em espessura 2 horas, os valores médios variaram de $0,29 \%$ a $0,66 \%$. Os painéis produzidos com $P$. oocarpa com relação madeira:cimento de 1:2,5 apresentaram médias estatisticamente iguais em comparação aos painéis de $P$. oocarpa com relação 1:2,5, $P$. caribaea com 1:3,0, P. maximinoi com 1:2,5 e 1:3,0, P. tecunumanii com 1:2,5 e 1:3,0 e, estatisticamente inferior em comparação aos painéis de demais tratamentos.

Para inchamento em espessura 24 horas, os valores médios variaram de $0,42 \%$ a $1,18 \%$. Os painéis produzidos com $P$. oocarpa com relação madeira:cimento de 1:2,5 apresentaram médias estatisticamente iguais em comparação aos painéis de $P$. oocarpa com relação 1:2,5, P. caribaea com 1:3,0, P. maximinoi com 1:2,5 e 1:3,0, P. tecunumanii com 1:2,5 e 1:3,0, P. taeda com 1:3,0 e, estatisticamente inferior em comparação aos painéis de demais tratamentos.

Em termos comparativos, os resultados de inchamento em espessura 24 horas, obtidos nesta pesquisa foram satisfatórios em comparação aos apresentados na literatura. Iwakiri et al. (2012) 
encontraram valores de IE 24 horas na faixa de 1,38\% a 1,95\% e de 0,39 a 0,92\%, respectivamente, para painéis produzidos com madeira de Schizolobium amazonicum e Cecropia hololeuca; Latorraca (2000) encontrou para painéis produzidos com Eucalyptus pellita, Eucalyptus robusta, Eucalyptus urophylla e Eucalyptus citriodora, valores na faixa de 1,64\% a 2,12\%. Já, Iwakiri et al. (2015) encontraram para painéis de Eucalyptus benthamii, valor médio de 0,79\%. Ressalta-se que, o maior valor obtido para as espécies de pinus tropicais foi de 1,04\%, que atendem às especificações de painéis comerciais produzidos pelo processo Bison (1978) para valores de inchamento em espessura 24 horas na faixa de $1,2 \%$ a $1,8 \%$.

Na tabela 2 estão apresentados os resultados da análise fatorial para o efeito das espécies estudadas.

Tabela 2. Resultados de massa específica aparente, absorção de água e inchamento em espessura - Análise fatorial para espécie.

Table 2. Results of density, water absorption and thickness swelling - Factor analysis for specie.

\begin{tabular}{lcccc}
\hline Espécie & AA 2 $\mathbf{h}$ (\%) & AA 24h (\%) & IE 2 h (\%) & IE 24h (\%) \\
P. taeda & $7,27 \mathrm{a}$ & $12,33 \mathrm{a}$ & $0,61 \mathrm{a}$ & $1,01 \mathrm{a}$ \\
& $(23,05)$ & $(14,63)$ & $(38,72)$ & $(39,83)$ \\
\hline \multirow{2}{*}{ P. maximinoi } & $8,40 \mathrm{ab}$ & $12,51 \mathrm{ab}$ & $0,44 \mathrm{~b}$ & $0,79 \mathrm{ab}$ \\
& $(24,21)$ & $(17,64)$ & $(56,30)$ & $(44,78)$ \\
\hline \multirow{2}{*}{ P. tecunumanii } & $8,13 \mathrm{ab}$ & $12,36 \mathrm{a}$ & $0,44 \mathrm{~b}$ & $0,77 \mathrm{~b}$ \\
& $(23,77)$ & $(16,27)$ & $(67,97)$ & $(48,40)$ \\
\hline \multirow{2}{*}{ P. oocarpa } & $8,58 \mathrm{~b}$ & $13,80 \mathrm{~b}$ & $0,36 \mathrm{~b}$ & $0,53 \mathrm{c}$ \\
& $(26,47)$ & $(19,23)$ & $(65,37)$ & $(56,57)$ \\
\hline \multirow{2}{*}{ P. caribaea } & $7,98 \mathrm{ab}$ & $12,23 \mathrm{a}$ & $0,54 \mathrm{ab}$ & $0,87 \mathrm{ab}$ \\
\end{tabular}

ME: massa específica aparente; AA: absorção de água; IE: inchamento em espessura; Médias seguidas de mesma letra na mesma coluna são estatisticamente iguais ao nível de probabilidade de $95 \%$. Valores entre parênteses referem-se ao coeficiente de variação.

Para absorção de água 2 horas, os painéis produzidos com madeira de $P$. taeda apresentaram média estatisticamente igual em comparação aos painéis de P. maximinoi, P. tecunumanii e P. caribaea e, estatisticamente inferior em comparação aos painéis de $P$. oocarpa. Resultados estatisticamente similares foram constatados para a absorção de água 24 horas. Portanto, com exceção de P. oocarpa, todas as demais espécies apresentaram comportamento satisfatório em relação aos painéis produzidos com a madeira de $P$. taeda, utilizada nesta pesquisa como espécie testemunha.

Para inchamento em espessura, tanto para 2 horas, quanto para 24 horas de imersão, as quatro espécies de pinus tropicais apresentaram médias estatisticamente iguais ou inferiores em comparação aos painéis testemunhas de $P$. taeda. Os melhores resultados para inchamento em espessura 2 horas foram obtidos para painéis de P. oocarpa, P. tecunumanii e P. maximinoi, e, para 24 horas, os melhores resultados foram obtidos para as espécies de $P$. oocarpa e P. tecunumanii.

Na tabela 3 estão apresentados os resultados da análise fatorial para o efeito das relações madeira:cimento.

Tabela 3. Resultados de massa específica aparente, absorção de água e inchamento em espessura - Análise fatorial para relação madeira:cimento.

Table 3. Results of density, water absorption and thickness swelling - Factor analysis for wood:cement ratio.

\begin{tabular}{lcccc}
\hline Relação madeira:cimento & AA 2 h (\%) & AA 24h (\%) & IE 2h (\%) & IE 24h (\%) \\
\multirow{2}{*}{$1: 2,5$} & 8,03 a & $12,93 a$ & $0,55 a$ & 0,95 a \\
& $(24,61)$ & $(17,32)$ & $(53,22)$ & $(39,72)$ \\
\hline \multirow{2}{*}{$1: 3,0$} & $8,11 \mathrm{a}$ & $12,36 \mathrm{a}$ & $0,41 \mathrm{a}$ & $0,64 \mathrm{a}$ \\
& $(22,54)$ & $(16,71)$ & $(61,43)$ & $(53,39)$ \\
\hline
\end{tabular}

ME: massa espećfica aparente; AA: absorção de água; IE: inchamento em espessura; Médias seguidas de mesma letra na mesma coluna são estatisticamente iguais ao nível de probabilidade de $95 \%$. Valores entre parênteses referem-se ao coeficiente de variação.

Os resultados da análise fatorial indicam que tanto para absorção de água, quanto para inchamento em espessura, não houve diferenças significativas entre os painéis produzidos com relação madeira:cimento de 1:2,5 e 1:3,0. Este resultado é bastante relevante sobre o ponto de vista econômico, tendo em vista a possibilidade de utilização de menor quantidade de cimento na composição dos painéis. Maior recobrimento das partículas com o cimento não influenciou significativamente na redução da absorção de água e inchamento em espessura. 
Iwakiri et al. - Potencial de uso de espécies de pinus tropicais para produção de painéis cimento-madeira

\section{Propriedades mecânicas dos painéis}

Na Tabela 4 estão apresentados os resultados de módulo de ruptura (MOR), módulo de elasticidade (MOE) e tração perpendicular (TP).

Tabela 4. Resultados de módulo de ruptura, módulo de elasticidade e tração perpendicular.

Table 4. Results of modulus of rupture, modulus of elasticity and perpendicular tension.

\begin{tabular}{lccc}
\hline Tratamento & MOR (MPa) & MOE (MPa) & TP (MPa) \\
P. caribaea 1:2,5 & $4,91 \mathrm{ab}$ & $2.920 \mathrm{abc}$ & $0,57 \mathrm{ab}$ \\
& $(16,32)$ & $(23,85)$ & $(15,43)$ \\
\hline P. caribaea 1:3,0 & $5,59 \mathrm{a}$ & $3.274 \mathrm{~b}$ & $0,85 \mathrm{a}$ \\
& $(14,97)$ & $(20,03)$ & $0,56 \mathrm{abc}$ \\
\hline P. maximinoi 1:2,5 & $5,53 \mathrm{a}$ & $2.729 \mathrm{abc}$ & $(18,98)$ \\
\hline P. maximinoi 1:3,0 & $(15,66)$ & $(23,89)$ & $0,64 \mathrm{a}$ \\
\hline P. oocarpa 1:2,5 & $5,08 \mathrm{ab}$ & $3.005 \mathrm{ab}$ & $(12,34)$ \\
\hline P. oocarpa 1:3,0 & $(11,10)$ & $(14,36)$ & $0,42 \mathrm{c}$ \\
\hline P. tecunumanii 1:2,5 & $4,69 \mathrm{bc}$ & $2.682 \mathrm{bc}$ & $(14,06)$ \\
\hline P. tecunumanii 1:3,0 & $(10,45)$ & $(13,12)$ & $0,62 \mathrm{a}$ \\
\hline P. taeda 1:2,5 & $5,37 \mathrm{a}$ & $3.300 \mathrm{a}$ & $(13,52)$ \\
\hline P. taeda 1:3,0 & $(12,86)$ & $(20,41)$ & $0,47 \mathrm{bc}$ \\
\hline
\end{tabular}

MOR: Módulo de ruptura; MOE: Módulo de elasticidade; TP: Tração perpendicular; Médias seguidas de mesma letra na mesma coluna são estatisticamente iguais ao nível de probabilidade de $95 \%$. Valores entre parênteses referem-se ao coeficiente de variação.

Os resultados da análise estatística das propriedades mecânicas dos painéis indicaram que houve diferenças significativas entre as médias dos tratamentos para módulo de ruptura, módulo de elasticidade e tração perpendicular.

Para o módulo de ruptura, os valores médios variaram de 3,90 MPa a 5,59 MPa. Os painéis de P. caribaea produzidos com relação madeira;cimento de 1:3,0 apresentaram média estatisticamente igual em comparação aos painéis de $P$. caribaea com relação 1:2,5, P. maximinoi com 1:2,5 e 1:3,0, $P$. oocarpa com 1:3,0 e, estatisticamente superior em comparação aos demais tratamentos.

Os resultados obtidos foram inferiores às especificações de painéis comerciais produzidos pelo processo Bison (1978) para valores mínimos de módulo de ruptura de 9,0 MPa. Com relação aos resultados apresentados na literatura, Latorraca (2000) obteve para painéis madeira:cimento de Eucalyptus pellita, Eucalyptus robusta, Eucalyptus urophylla e Eucalyptus citriodora, valores de MOR entre 3,53 MPa a 6,77 MPa. Iwakiri e Prata (2008) encontraram para painéis de Eucalyptus dunnii, valores entre 3,53 MPa a 4,02 MPa. Iwakiri et al. (2015) encontraram para painéis de Eucalyptus benthamii, valor médio de 5,27 MPa.

Para o módulo de elasticidade, os valores médios variaram de $1.943 \mathrm{MPa}$ a $3.300 \mathrm{MPa}$. Os painéis de P. oocarpa produzidos com relação madeira:cimento de 1:3,0 apresentaram média estatisticamente igual em comparação aos painéis de $P$. maximinoi com relação 1:2,5 e 1:3,0, P. tecunumanii com 1:2,5 e 1:3,0 e estatisticamente superior em comparação aos demais tratamentos.

Com relação a outras pesquisas publicadas, os resultados de MOE obtidos para espécies de pinus tropicais estão próximos dos apresentados por Latorraca (2000) para quatro espécies de eucalipto - Eucalyptus pellita, Eucalyptus robusta, Eucalyptus urophylla e Eucalyptus citriodora, cujos valores foram de $1.840 \mathrm{MPa}$ a $3.390 \mathrm{MPa}$. Iwakiri et al. (2012) encontraram para painéis madeira-cimento de Schizolobium amazonicum e Cecropia hololeuca, valores de MOE de $2.571 \mathrm{MPa}$ e $2.836 \mathrm{MPa}$, respectivamente. Iwakiri et al. (2015) encontraram para painéis de Eucalyptus benthamii, valor médio de MOE de $2.854 \mathrm{MPa}$. Os painéis de $P$. caribaea produzidos com relação madeira:cimento de 1:3,0, $P$. maximinoi com relação 1:3,0 e P. oocarpa com 1:3,0 foram os que atenderam ao requisito mínimo de 3.000 MPa estabelecido pelo processo Bison (1978) para painéis comerciais de madeira:cimento.

Para a tração perpendicular, os valores médios variaram de $0,41 \mathrm{MPa}$ a $0,85 \mathrm{MPa}$. Os painéis de P. caribaea produzidos com relação madeira:cimento de 1:3,0 apresentaram média estatisticamente igual em comparação aos painéis de P. caribaea com relação 1:2,5, P. maximinoi com 1:2,5 e 1:3,0, 
P. oocarpa com 1:3,0, P. tecunumanii com 1:3,0, P. taeda com 1:3,0 e, estatisticamente superior em comparação aos demais tratamentos.

Na comparação com os dados apresentados na literatura, os resultados obtidos para as espécies de pinus tropicais foram satisfatórios aos valores médios obtidos por Iwakiri e Prata (2008) para painéis madeira:cimento de Eucalyptus grandis na faixa de 0,24 MPa a 0,47 MPa; aos resultados obtidos por Okino et al. (2004) para painéis de Hevea brasiliensis na faixa de 0,27 MPa 0,43 MPa; e, aos resultados apresentados por Santos et al. (2008) para painéis produzidos com madeira de Pinus taeda com misturas de 25, 50 e 75\% de Eremanthus erythropappus, de 0,60 MPa, 0,68 MPa e 0,54 $\mathrm{MPa}$, respectivamente. Todos os resultados de tração perpendicular obtidos nesta pesquisa atenderam ao requisito mínimo de 0,40 MPa estabelecido pelo Processo Bison para painéis comerciais de madeira:cimento.

Na tabela 5 estão apresentados os resultados da análise fatorial para o efeito das espécies estudadas.

Tabela 5. Resultados de módulo de ruptura, módulo de elasticidade e tração perpendicular - Análise fatorial para espécie.

Table 5. Results of modulus of rupture, modulus of elasticity and perpendicular tension - Factor analysis for specie.

\begin{tabular}{lccc}
\hline Espécie & MOR (MPa) & MOE (MPa) & TP (MPa) \\
P. caribaea & $5,25 \mathrm{a}$ & $3.088,80 \mathrm{a}$ & $0,72 \mathrm{a}$ \\
& $(17,67)$ & $(23,18)$ & $(23,92)$ \\
\hline \multirow{2}{*}{ P. maximinoi } & $5,30 \mathrm{a}$ & $2.874,53 \mathrm{a}$ & $0,60 \mathrm{~b}$ \\
& $(13,38)$ & $(22,55)$ & $(17,90)$ \\
\hline \multirow{2}{*}{ P. oocarpa } & $5,03 \mathrm{a}$ & $3.024,06 \mathrm{a}$ & $0,54 \mathrm{~b}$ \\
& $(15,48)$ & $2.868,00 \mathrm{a}$ & $(25,61)$ \\
\hline \multirow{2}{*}{ P. tecunumanii } & $4,53 \mathrm{~b}$ & $(23,24)$ & $0,51 \mathrm{~b}$ \\
\hline \multirow{2}{*}{ P. taeda } & $(12,54)$ & $2.200,92 \mathrm{~b}$ & $(21,46)$ \\
\hline
\end{tabular}

MOR: Módulo de ruptura; MOE: Módulo de elasticidade; TP: Tração perpendicular; Médias seguidas de mesma letra na mesma coluna são estatisticamente iguais ao nível de probabilidade de $95 \%$. Valores entre parênteses referem-se ao coeficiente de variação.

Os painéis produzidos com madeira de P. caribaea, P.maximinoi e P. oocarpa apresentaram valores médios de MOR, estatisticamente iguais entre si e superiores aos painéis de $P$. tecunumanii e $P$. taeda. Para o MOE, todas as quatro espécies de pinus tropicais apresentaram médias estatisticamente iguais entre si e superiores em comparação aos painéis de $P$. taeda. Já, para a tração perpendicular, os painéis de $P$. caribaea apresentaram média estatisticamente superior em comparação aos painéis de demais espécies. É importante observar que, para as três propriedades mecânicas avaliadas, os painéis produzidos com todas as espécies de pinus tropicais apresentaram melhores resultados em comparação aos painéis testemunhas, produzidos com madeira de P. taeda.

Na tabela 6 estão apresentados os resultados da análise fatorial para o efeito das relações madeira:cimento.

Tabela 6. Resultados de módulo de ruptura, módulo de elasticidade e tração perpendicular - Análise fatorial para relação madeira:cimento.

Table 6. Results of modulus of rupture, modulus of elasticity and perpendicular tension - Factor analysis for wood:cement ratio.

\begin{tabular}{lccc}
\hline Relação madeira:cimento & MOR $(\mathbf{M P a})$ & MOE (MPa) & TP (MPa) \\
\multirow{2}{*}{$1: 2,5$} & $4,70 \mathrm{a}$ & $2.612,73 \mathrm{~b}$ & $0,47 \mathrm{~b}$ \\
& $(17,33)$ & $(26,26)$ & $(22,70)$ \\
\hline \multirow{2}{*}{$1: 3,0$} & $5,00 \mathrm{a}$ & $3.009,79 \mathrm{a}$ & $0,68 \mathrm{a}$ \\
\hline
\end{tabular}

MOR: Módulo de ruptura; MOE: Módulo de elasticidade; TP: Tração perpendicular; Médias seguidas de mesma letra na mesma coluna são estatisticamente iguais ao nível de probabilidade de $95 \%$. Valores entre parênteses referem-se ao coeficiente de variação.

Os resultados da Tabela 6 indicam que para o MOE e tração perpendicular, os painéis produzidos com maior relação madeira:cimento $(1: 3,0)$ apresentaram médias estatisticamente superiores em comparação aos painéis produzidos com menor relação madeira:cimento $(1: 2,5)$. Para o MOR, não foram constatadas diferenças significativas entre as médias obtidas para relações madeira:cimento de 1:3,0 e 1:2,5. Valor intermediário entre as duas relações madeira:cimento testadas, podem ser avaliadas para obter melhor relação custo benefício. 
Iwakiri et al. - Potencial de uso de espécies de pinus tropicais para produção de painéis cimento-madeira

\section{CONCLUSÕES}

As quatro espécies de pinus tropicais apresentaram menores valores de inchamento em espessura em comparação aos painéis testemunhas de $P$. taeda. $\mathrm{O}$ aumento na relação madeira:cimento não afetou de forma significativa as propriedades de absorção de água e inchamento em espessura.

Os painéis produzidos com as quatro espécies de pinus tropicais apresentaram médias das propriedades mecânicas estatisticamente iguais ou superiores em comparação aos painéis testemunhas de P. taeda.

O aumento na relação madeira:cimento não afetou de forma significativa os resultados de módulo de ruptura, mas contribuiu para aumento nos valores de módulo de elasticidade e tração perpendicular.

O inchamento em espessura e tração perpendicular, que são duas importantes propriedades de painéis cimento-madeira, atenderam as especificações para painéis comerciais produzidos pelo processo Bison.

Com base nas avaliações comparativas com os resultados obtidos para a espécie testemunha e dados apresentados na literatura, pode-se afirmar que as espécies de P. caribaea, P. maximinoi, $P$. oocarpa e P. tecunumanii, são potencialmente viáveis para produção de painéis cimento-madeira.

\section{AGRADECIMENTOS}

Os autores expressam seus agradecimentos, à empresa Valor Florestal S/A, pela doação das madeiras utilizadas nesta pesquisa.

\section{REFERÊNCIAS BIBLIOGRÁFICAS}

BERALDO, A. L.; ARRUDA, A. C.; STANCATO, A. C.; SAMPAIO, C. A. P; FERNANDES FILHO, O. P.; LEONEL, V. M. Compósito à base de resíduos vegetais e cimento portland. In: ENCONTRO BRASILEIRO EM MADEIRAS E ESTRUTURAS DE MADEIRA. Uberlândia. 8., 2002, Uberlândia. Anais... Uberlândia: EBRAMEM, 2002. p. 85-90.

BISON. Wood-cement board. Berlin: Springer, 1978. 10 p.

CASTRO, V. G.; BRAZ, R. L.; AZAMBUJA, R. R.; LOIOLA, P. L.; IWAKIRI, S.; MATOS, J. L. M. Painéis cimentomadeira de Eucalyptus saligna com diferentes aditivos químicos e métodos de formação. Floresta, Curitiba, v. 45, n. 2, p. 349-360, 2015.

EN EUROPEAN COMMITTEE FOR STANDARDIZATION. EN 317: Determination of swelling in thickness after immersion in water. Bruxelas, 1993a. 5 p.

EUROPEAN COMMITTEE FOR STANDARDIZATION. EN 319: Determination of tensile strength perpendicular to the plane of the board. Bruxelas, 1993b. 7 p.

EN - EUROPEAN COMMITTEE FOR STANDARDIZATION. EN 310: Determination of modulus of elasticity in bending and bending strength. Bruxelas, 1993c. $10 \mathrm{p}$.

IWAKIRI S; PRATA, J. G. Utilização da madeira de Eucalyptus grandis e Eucalyptus dunnii na produção de painéis de cimento-madeira. Cerne, Lavras, v. 14, n. 1, p. 75-81, 2008.

IWAKIRI, S.; TRIANOSKI, R.; CUNHA, A.B.; PRATA, J.G.; HARA, M.; BILA, N.F.; GUINA LUIS, R.C.; ARAÚJO, R.D. Propriedades tecnológicas de painéis cimento-madeira produzidos com partículas de eucalipto. Revista de Ciências Agroveterinárias, Lages, v. 14, n. 3, p. 217-223, 2015. 
IWAKIRI S.; SILVA, L. S.; TRIANOSKI, R.; BONDUELLE, G. M.; ROCHA, V. Y. Avaliação do potencial de utilização da madeira de Schizolobium amazonicum "paricá" e Cecropia hololeuca "embaúba" para produção de painéis cimento-madeira. Cerne, Lavras, v. 18, n. 2, p. 303-308, 2012.

LATORRACA, J. V. F. Eucalyptus spp na produção de painéis cimento madeira. 2000. 191 p. Tese (Doutorado em Ciências Florestais) - Universidade Federal do Paraná, Curitiba, 2000.

MOSLEMI A, A. Particleboard. London: Southern Illinois University, 1974. 245 p.

MOSLEMI, A. A.; GARCIA, J. F.; HOFSTRAND, A. D. Effect of various treatments and additives on WoodPortland cement-water systems. Wood and Fiber Science, New York, v. 15, n. 2, p. 164-176, 1983.

OKINO E. U. A.; SOUZA, M. R.; SANTANA, M. A. E.; SOUSA, M. E.; TEIXEIRA, D. E. Chapa aglomerada de cimento-madeira de Hevea brasiliensis Müll. Arg. Revista Árvore, Viçosa, v. 28, n. 3, p. 451-457, 2004.

PARCHEN, C. F. A.; IWAKIRI, S.; ZELLER, F.; PRATA, J. G. Vibro-dynamic compression processing of low density wood-cement composites. European Journal of Wood Prod, Berlin, v. 11, n. 1, p. 01-09, 2015.

RASHWAN M. S.; HATZINICOLAS, M.; ZMAVC, R. Development of a lightweight low-cost concrete block using Wood residues. Forest Products Journal, Amsterdam, v. 42, n. 5, p. 57-64, 1992.

SANTOS, R. C.; MENDES, L. M.; MORI, F. A.; MENDES, R. F. Aproveitamento de resíduos da madeira de candeia (Eremanthus erythropappus) para produção de painéis cimento-madeira. Cerne, Lavras, v. 14, n. 3, p. 241-250, 2008.

SILVA, G. C.; LATORRACA, J. V. F; TEIXEIRA, D. E.; BORTOLETTO JUNIOR, G. Produção do compósito madeira e casca de Eucalyptus urophylla S.T. Blake e cimento Portland. Scientia Forestalis, Piracicaba, n. 68, p. 59-67, 2005.

SIMATUPANG , M. H.; SCHWARZ, G. H.; BROOKER, F. W. Small scale plants for the manufacture of mineralbonded wood composites. In: World Forestry Congress. Jakarta: WIC, 1978, 21 p.

Recebido em 29/03/2016

Aceito para publicação em 05/10/2016

Sci. For., Piracicaba, v. 45, n. 113, p. 151-159, mar. 2017 DOI: dx.doi.org/10.18671/scifor.v45n113.15 